\title{
Agroindustrial complex system: methods of obtaining, analysis and processing of expert information
}

\author{
Alla Rasputina* \\ Baikal state University, Irkutsk, Russia
}

\begin{abstract}
Methods for obtaining, analyzing and processing expert information in the system of the agro-industrial complex are of great and ever increasing importance. Moreover, the subsystem of expert information is constantly evolving as a result of the qualitative growth of information and communication technologies and the improvement of the methodological and methodological apparatus of organizing and conducting examinations. Nevertheless, the potential and aggregate possible subsystems of expert information are not used effectively enough due to various reasons, both objective and subjective. The main purpose of the expert information subsystem is to organize and conduct examinations that ensure a high professional level of decision-making in the system of the agro-industrial complex at different levels of management. The research methodology is based on the selective application of system analysis methods aimed at studying complex objects and processes of the agro-industrial complex system, with the predominant use of expert assessment methods. In the process of the first stage of the study of the expert and information subsystem of the agro-industrial complex, based on the method of passive examination in the context of the agricultural digitalization project, a digraph of the structure of the problem field of digitalization was developed, which clearly reflects the interconnections and interactions of individual structural elements of the agro-industrial complex. A scheme for a multistage examination of the problem of digitalization of the regional system of the agro-industrial complex has been developed, an algorithm has been developed for the subsystem of expert information of the agro-industrial complex in the form of a tree-like digraph, reflecting the functional structure of the subsystem. This will allow in the future, with the active use of the developed algorithm for the optimal functioning of the subsystem of expert information of the agro-industrial complex, to ensure high efficiency of the decisions made and the transition to cognitive models of system control.
\end{abstract}

\footnotetext{
${ }^{*}$ Corresponding author: rasp77@mail.ru
} 


\section{Introduction}

The system of agro-industrial complex (hereinafter - AIC) refers to complex heterogeneous systems. It includes three subsystems of different nature and functioning based on different laws: economic, technical, environmental, and social. As a rule, the effect of these laws is manifested in the form of even more complex interpenetrating inter-system formations: socio-technical, socio-economic, environmental-economic, technicaltechnological, natural-technical.

At the present stage of social development, expert evaluations play one of the key roles in almost all branches of knowledge. The method of expert evaluation is the most effective method for solving problems in probabilistic systems. Expert assessments are most often used in situations where information cannot be fully formalized $[29,10]$.

The main purpose of organizing and conducting expert reviews is to improve the professional level of decisions made by using specially developed and proven in practice technologies of expert review.

The examination of complex systems must be specially organized. The problems should be dissected, broken down into a sequence of separate, simple questions, already accessible to the competence of the experts. It is as if the expert plays the role of some device that either makes a preferred choice, or sets the value of the coefficients, or connects cause and effect by a logical thread.

The staging of expert interviews is essential in obtaining expert information $[3,14,2]$. In some situations, it may be most appropriate to develop new peer review technology [15].

A questionnaire in expert methods is a set of questions organized in a certain way, the answers to which are considered as information about the degree of confidence of the expert in the probability of occurrence of events or in the relative importance of the assessed property.

Since expert methods are also largely based on the use of questionnaires, much attention should be paid to the organization of this procedure $[3,4]$. In the first stage of peer review, a passive peer review method is often used.

Passive expertise consists of expert monitoring of print and electronic media and other online resources (social networks, databases, the content of forwarded information, etc.) according to a certain set of criteria, indicators with subsequent analytical processing [8].

A matrix of pairwise comparisons is often used in the analysis and processing of expert information. The matrix of pairwise comparisons is the main tool used in the method of pairwise comparison of criteria or alternatives, as well as in the method of hierarchy analysis. It is widely used in decision-making tasks, being a well-studied, simple, and understandable technique for experts, especially for many criteria [34].

In recent years, there has been an increase in the importance of and opportunities for conducting networked exposures to support management decisions. Network expertise can also be based on cognitive modeling and hierarchy analysis [28, 29].

In the AIC system, as in other large complex systems of heterogeneous nature, numerous and diverse expert evaluation methods are widely used. The appropriateness of a particular method is determined by the nature of the analyzed information [2, 19, 20, 22].

There are a certain number of specific problems related to internal contradictions and the impact of the external environment in the agro-industrial complex - all of them, to varying degrees, have been studied and identified for more in-depth study to develop effective algorithms of production and management activities [11,12,16,17].

Many works consider the functioning and interaction of subsystems of agroindustrial complex, technological and social processes based on system-cognitive approach, with active use of formal and heuristic methods. 
Expert assessments are an established scientific method for the analysis of complex nonformalizable problems [22].

The adequacy of the mathematical description to the studied dynamic process, as a rule, is debatable - always based on some postulates, hypotheses [3,4,13,19,20].

Most authors who study expert evaluation methods that are as application-oriented as possible are inclined to believe that in many management problems, the simplicity of the mathematical apparatus is often a more important circumstance than the perceived accuracy of the results $[3,4,13,19,25-27,30]$.

For example, B.G. Litvak, a renowned expert on expert methods, believes that one of the typical mistakes when using expert information for the preparation of management decisions includes an unjustified preoccupation with formal models [15].

Therefore, heuristic or informal methods used with experts are of particular relevance [5$6]$.

Informal systems analysis methods mobilize the intuition and expertise of professionals and are used constructively to address the organization of analytical activities. Informal systems analysis methods mobilize the intuition and expertise of specialists and are used constructively on the solution of analytical activity organization [7,35,37].

In order to obtain realistic results, it is important to adequately perceive the complexity of the situations being investigated. In this respect, the method of hierarchy analysis (MAI) developed by Saaty is very convenient in expert analysis. Different opinions and conflicts are tolerated, as they are in the real world.

In the hierarchy analysis method (as in the more advanced analytic network method (ANM)), a matrix of pairwise comparisons can be generated based on any relationship scale applied to the measurable properties of the objects being compared. The comparison can be made based on actual measurements or a fundamental scale that reflects the relative strength of preference and feeling [31,32,36,38].

The result of many expert examinations is a ranking of the objects of expertise according to some criterion, attribute, or property. The pairwise comparisons method involves obtaining information from an expert only about the comparative preference of one of the two objects presented [15].

The set of group preferences (examination results) is represented in the form of a) ordinal (ordinal) preference - here the objects are ordered by rank or place in the general population, b) cardinal (numerical) preference - here each factor is assigned a weight or number Wi, the entire group of evaluated factors is described by a vector:

$$
\mathrm{W}=\left(\begin{array}{c}
W_{1} \\
\cdot \\
\cdot \\
W_{i} \\
\cdot \\
\cdot \\
W_{n}
\end{array}\right) \text {, usually } \sum_{i=1}^{n} w_{i}=1
$$

In the normalization method, each expert Ej assigns a certain score Pij to Fi (e.g., Pij $\in[0$, 1]). It is convenient to set the scores on the questionnaire as shown below: 


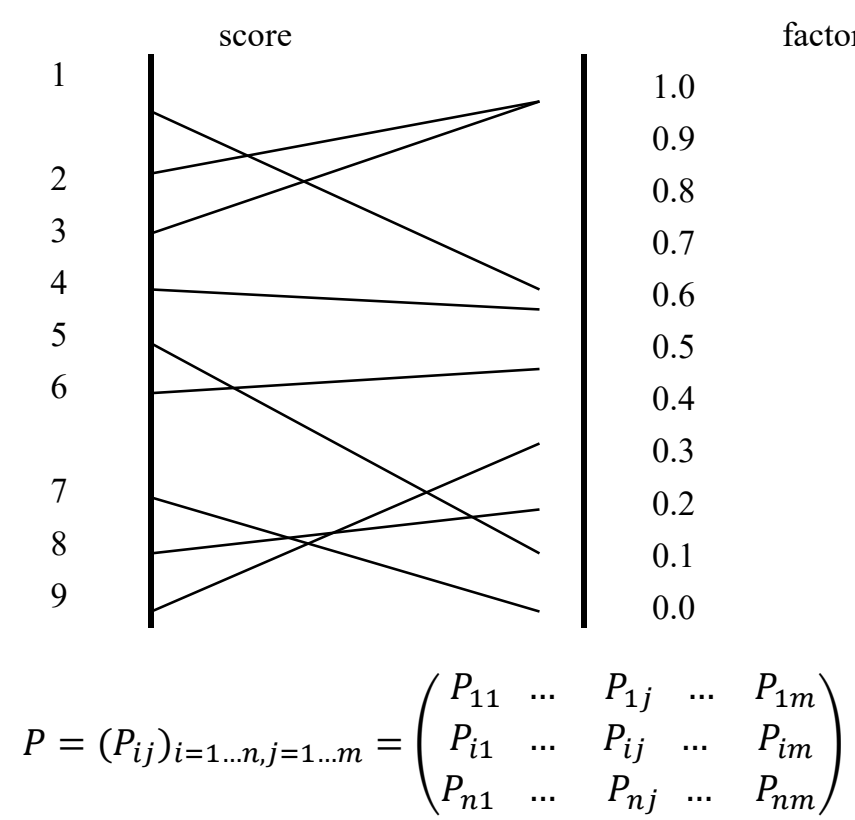

$P_{i j}$ - the score assigned to the $\mathrm{i}$-th factor from the point of view of the $\mathrm{j}$-th interviewee

Based on the matrix, $P=\left(P_{i j}\right)_{i=1 \ldots n, j=1 \ldots m}$ the elements of matrix $\mathrm{X}$ are defined by normalizing to one:

$$
X_{i j}=\frac{P_{i j}}{\sum_{i=1}^{n} P_{i j}}
$$

The resulting group opinion in the ordinal representation in the analysis phase is assessed - can the results be trusted? At different configurations, it is only possible to estimate the density of the opinion area quantitatively, to estimate its dimensions and distance between vectors, and from the centroid of the area to each of the vectors, further using methods of probability theory [23].

According to the increase or decrease of this attribute, ranking by some attribute is the arrangement of objects in a numbered sequence. The rank xi indicates the place that the $i$-th object occupies in this sequence. If $m$ experts rank $n$ objects, we get a ranking matrix, which lists experts by rows and objects by columns. At the intersection of rows and columns are written values xij - the rank of the $\mathrm{i}$-th object assigned to it by the $\mathrm{j}$-th expert.

To assess the degree of consistency of experts' opinions on the ranking of objects, a coefficient of concordance $\mathrm{W}$ is used, which varies from 0 to 1 :

$$
\mathrm{W}=\frac{12 \sum_{i=1}^{i=n}\left[\sum_{i=1}^{i=m}\left(\mathrm{x}_{\mathrm{ij}}\right)-0.5 \mathrm{~m}(\mathrm{n}+1)\right]^{2}}{m^{2}\left(n^{3}-n\right)}
$$

If the experts' opinions are well-coordinated, and they all gave the objects exactly the same ranking, then the concordance coefficient $W=1$; if $W=0$ there is no connection between the experts' rankings, i.e., their opinions completely diverge [18].

It is not always necessary to strive for all experts to come to the same opinion (and often it is impossible) - the diversification and diversity of opinions is a positive property of expertise - it allows to consider the subject of expertise comprehensively and find unexpected opportunities for solutions [8]. 


\section{Methodology}

The methodology of our research is based on the selective application of system analysis methods aimed at studying the complex objects of the agro-industrial complex system, with the predominant use of expert evaluation methods.

In addition to the methods of expert assessments in the entire wide range, we consider it expedient to use methods of scenario forecasting, as well as methods of cognitive analysis and modeling, which we propose to implement at the next stages of the study.

\section{Results}

During the first stage of the study of the expert-information subsystem of the AIC, based mainly on the method of passive expertise, first of all - the study of a certain sample of expert publications on the state of problems occurring in the AIC system in the context of the digitalization project, an organizational chart of the problem field of digitalization of agriculture, visually reflecting the relationship and interaction of individual structural elements, was developed:

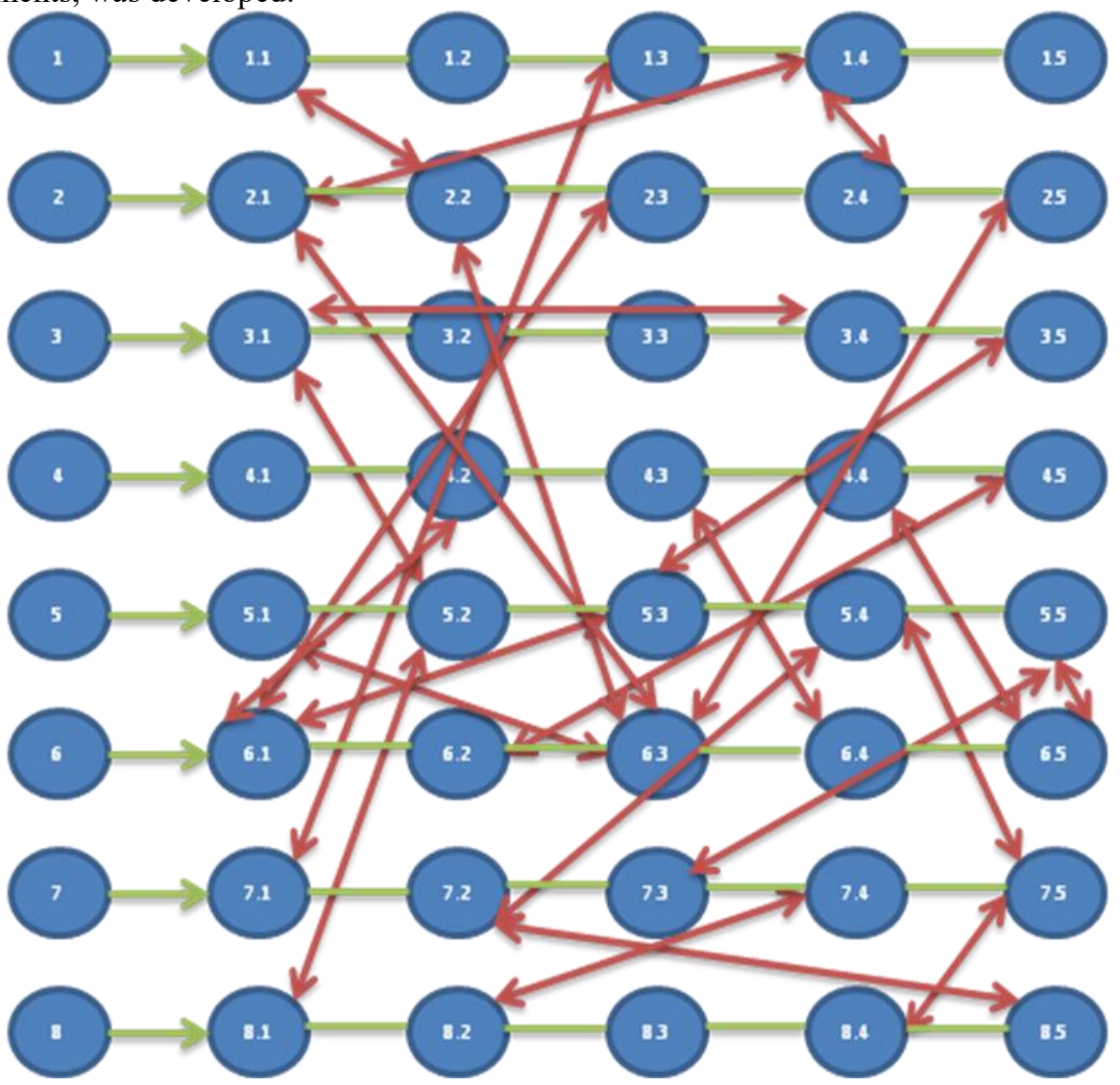

Fig. 1. Orgraph of the structure of the problem field of digitalization in the agroindustrial complex system.

1 - Organizational and Economic Characteristics of Agribusiness; 2 - Agrotechnologies; 3 - Innovation in Agribusiness; 4 - The Human Factor; 5 - Digitalization; 6 - Planning, Modeling, Forecasting; 7 - Experts and Consultants; 8 - Training IT-technologies. 
To further analyze the results of passive expertise, the scheme of multistage expertise of the problem of digitalization of the regional system of the agro-industrial complex was developed

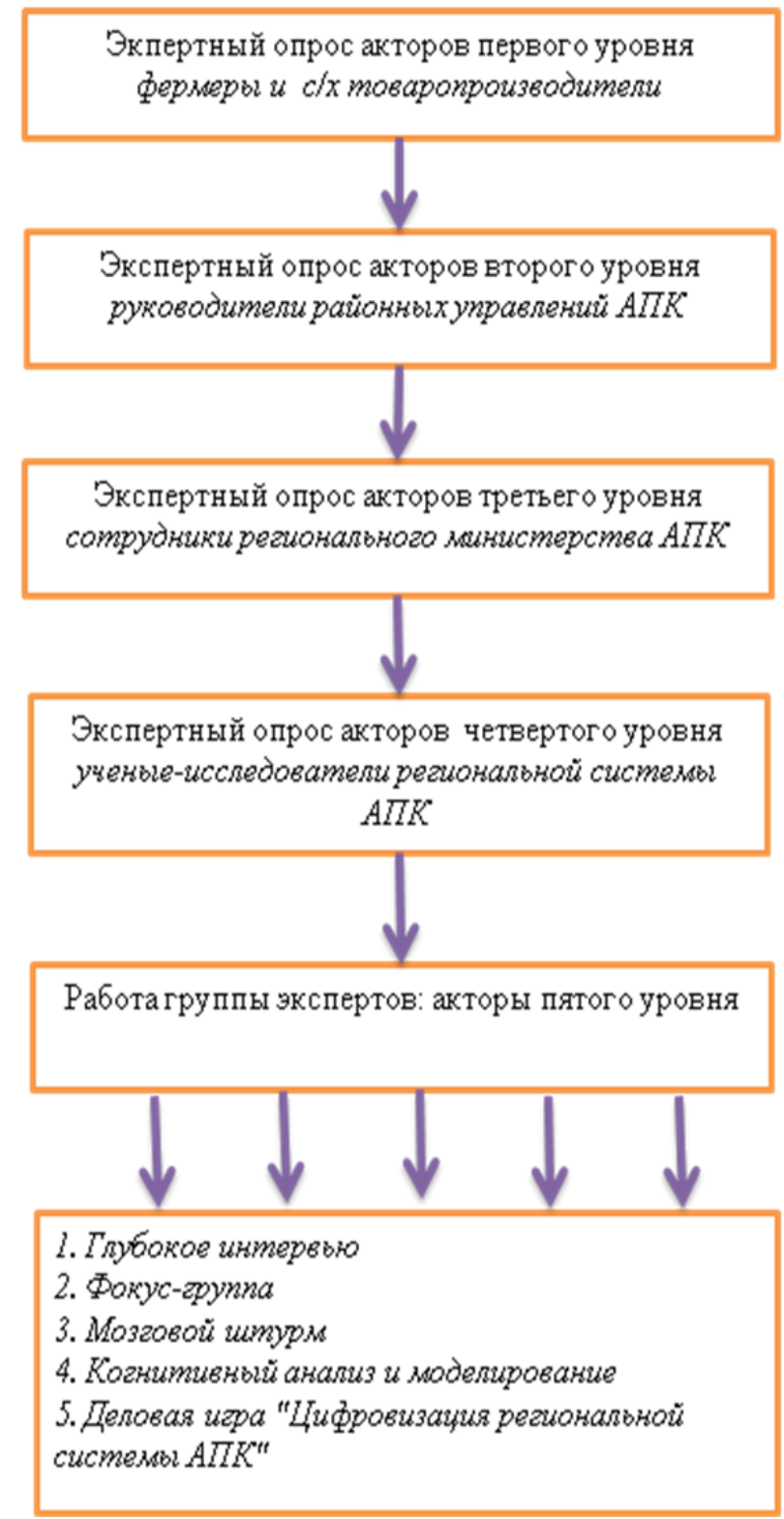

Fig 2. Scheme of multistage examination of the problem of digitalization of the regional system of agroindustrial complex.

For the further successful solution of the research tasks of the object - AIC system, the algorithm of the AIC subsystem of expert information in the form of a tree-structured orgraph reflecting the functional structure of the subsystem was formulated: 


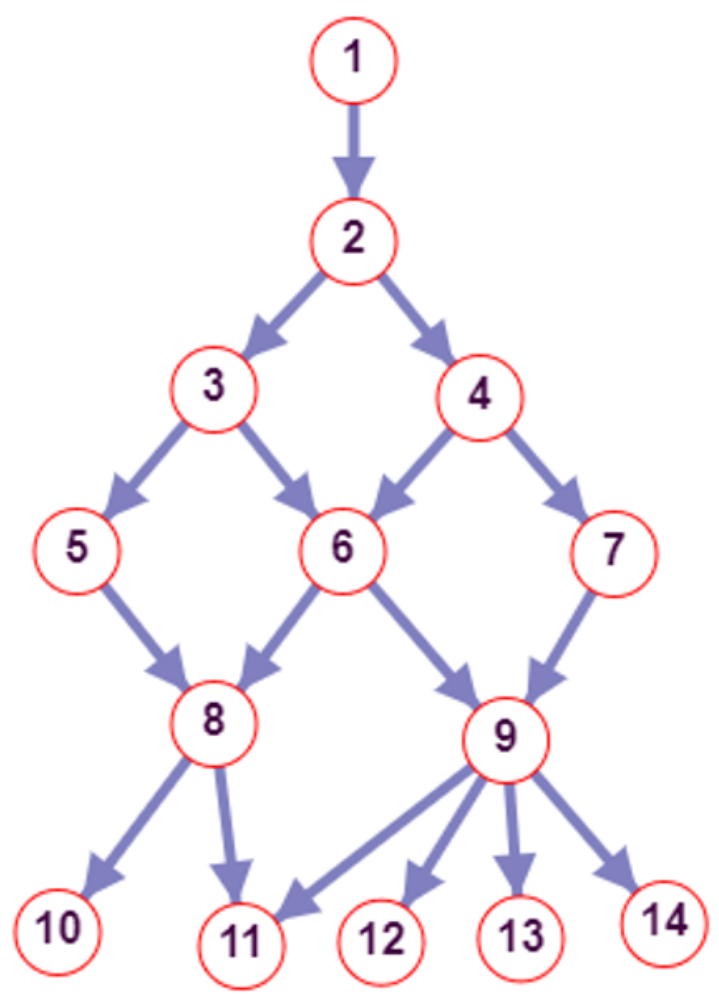

Fig. 3. A subsystem of expert information of the AIC (structural and functional mapping).

1 - Decision Maker (DM), 2 - Decision Making Process, 3 - Ministry of Agriculture Experts, 4 - Expert Systems, 5 - Information and Consultation Service, 6 - Passive Expertise 7 - Network expertise, 8 - Local expertise, 9 - Big problem expertise, 10 - Brainstorming method, 11 - Development scenarios, 12 - Focus group, in-depth interview, 13 - Business and simulation games, 14 - Cognitive models and technologies

\section{Discussion of results}

It should be borne in mind that the lack of a quality approach to the organization and management in the preparation and direct conduct of the examination, as well as in the further analysis of the information obtained, can significantly reduce its effectiveness. This is especially true of collective examinations. Mathematical and statistical methods of expert information processing are least applied at the preparation and direct conduct of expert interviews (Abaev, 2012).

Most of the developed formal models do not find application in the practice of the AIC system due to the lack of qualitative description in natural language.

Expert judgment methods have a considerable variety and are relatively rarely used in forecasting theory and practice. Cognitive modeling techniques combine well with expert judgment techniques.

Cognitive analysis and modeling technology make it possible to explore different problems with clear and fuzzy factors and relationships, taking into account the influence of the external environment, to predict the development of emerging situations, resulting in a 
manageable cognitive process used to support managerial decision-making (Sukhodolov et al., 2018).

Only when there is a clear goal, and a decision-making algorithm is the meaning, value, and meaning of the information fully revealed. In addition, the evaluation of the information is subjective in nature, as it depends on the subject's ability to use it" (Moiseev, 1995).

\section{Conclusions}

The problem field of digitalization of agriculture obtained as a result of our research, expressed in tabular and graphical form, is a prototype of a cognitive map, convenient for further use when working with experts in the agro-industrial complex. Two digitalization scenarios will be developed using expert assessment methods: pessimistic and realistic. The algorithms of the above scenarios (especially for the weak points of the optimistic scenario) can be of practical importance for use in the decision-making process on a digitalization project at all hierarchical levels of management in the agro-industrial complex system.

There is a need for the active use of expert assessment methods when discussing a departmental project for the digitalization of agriculture. This will identify weaknesses or critical paths in the optimistic scenario presented by the Ministry of Agriculture.

At the subsequent stages of the study, the optimal algorithms for a realistic digitalization scenario are expected to be developed by means of expert evaluation methods.

The active use of the entire range of methods for expert assessment of predicted and simulated situations, the use of cognitive analysis and modeling in the development and implementation of a scenario for the digitalization of agriculture can be of decisive importance in this process.

Given the informalizability of individual stages and structural elements of complex systems when using the procedures of system analysis, it is necessary to use more actively the methods of expert evaluations, prediction scenarios, structural-schematic models of verbal nature based on cognitive analysis, and modeling.

When modeling the socio-techno-natural systems of the agro-industrial complex, it is advisable to use expert evaluations and methods of hierarchy analysis.

From this, we can conclude: there are as many subjects as subjective conceptions of the object or as many subjective models of the object, not counting that any subject can create not one but two or three or more models of the same object. Further, as a consequence, there are as many experts as there are expert systems in a latent state, accessible only for use by the expert carrier of exclusive and unique in its own way information.

In the case of exteriorization and formalization of such a unique expert system belonging to a single entity, it becomes a formalized expert system, an information product available for use by an indefinite number of consumers - users of a particular software product.

\section{References}

1. L. Ch. Abaev, Problems of National Strategy 6(15), 29 (2012)

2. A. N. Anokhin, Methods of peer review: a textbook (1996)

3. S.D. Beshelev, F.G. Gurvich, Expert Evaluations (1973)

4. S.D. Beshelev, F.G. Gurvich, Mathematical and statistical methods of expert judgment (1980)

5. S.A. Belanovsky, Deep interview (2001a)

6. S.A. Belanovsky, Focus group method (2001b)

7. R.G. Bolbakov, State Councilor 1(9), 45 (2015) 
8. D.A. Gubanov, N.A. Korgin, D.A. Novikov, A.P. Raikov, Network Expertise, 2nd ed. (2011)

9. V.A. Zhuravleva, Bulletin of RUDN University, Series: Sociology 2, 28 (2012)

10. V.A. Zhuravleva, Expert assessment in solving cognitive problems of knowledge societies: theoretical and methodological principles: author ... dissertation ... cand. social sciences, special 22.00.01 (2013)

11. Ya.M. Ivagno, Extreme Natural Events: Methodology, Modeling and Forecasting (2007)

12. V.N. Kurochkin, M.V. Grechko, Bulletin of Agrarian Science of the Don 37, 25 (2017)

13. O.I. Larichev, Theory and Methods of Decision Making, and a Chronicle of Events in Fairy Lands (2000)

14. B.G. Litvak, Expert Information: Methods of Derivation and Analysis (1982)

15. B.G. Litvak, Expert Judgment and Decision Making (1996)

16. L.M. Lukyanova, Bulletin of the Russian Academy of Sciences, Theory and control systems 5, 100 (2007)

17. E.V. Lutsenko, Polythematic network electronic scientific journal of the Kuban State Agrarian University 83, 695 (2012)

18. A.G. Mamikonov, Decision Making and Information (1983)

19. N.N. Moiseev, The Simplest Mathematical Models of Economic Forecasting (1975)

20. N.N. Moiseev, Mathematical Problems of Systems Analysis (1981)

21. N.N. Moiseev, Modern Rationalism (1995)

22. A.N. Pavlov, B.V. Sokolov, Methods of processing expert information: a training manual (2005)

23. T.L. Partyka, I.I. Popov, Mathematical methods: textbook. 2nd ed., Rev. and add. (2007)

24. T.G. Arbatskaya, Ya. V. Putyrskaya, Problems of recognizing the costs of restoring real estate by organizations of the general government sector Audit statements 1, 14 (2019)

25. N.V. Polyakova, V.V. Polyakov, V.S. Kryazhev, A.P. Kryazheva, Russian Journal of Criminology 12(3), 330 (2018)

26. A. Rasputina, N. Zhilkina, S. Ovanesyan, V. Tyunkov, FRED-2019, 01.113 (2020)

27. A.V. Rasputina, O. Yu Ziboreva, Economics Business and Management Research 81, 331 (2019)

28. A.N. Raikov, UBS, 30-1, 758 (2010)

29. A.N. Raikov, Defense Complex - to the Scientific and Technological Progress of Russia 3(127), 3 (2015)

30. G. Rademacher, O. Teplitz, Numbers and Figures (1962)

31. T. Saaty, K. Kerns, Analytical Planning. Organization of Systems (1991)

32. T.L. Saati, Decision Making with Dependencies and Feedback Loops. Analytical networks (2008)

33. Yu.V. Sidelnikov, Systems analysis of expert forecasting (2007)

34. V.A. Sudakov, MLSD'2018, 526 (2018)

35. A.P. Sukhodolov, M.A. Kaluzhina, B.A. Spasennikov, V.S. Kolodin Russian Journal of Criminology 13(3), 385 (2019)

36. V.I. Samarukha, A.V. Samarukha, I.V. Samarukha, Russian Journal of Criminology, 12(2), 199 (2018) 
37. A.P. Sukhodolov, A.P. Fedotov, M.M. Makarov, P.N. Anoshko, P.G. Sorokina, A.V. Kolesnikova, D.A. Zhabina Bulletin of Baikal State University 30(2), 233 (2020)

38. E. Khitrova, T. Khitrova, Economics Business and Management Research 81,312 (2019) 